Y.-H. Chu, N.B. Suntzeff, J.E. Hesser, and D.A. Bohlender, eds.

\title{
The Star Formation History of the Magellanic Clouds: A Preliminary Report
}

\author{
Jason Harris, Dennis Zaritsky and Eva K. Grebel \\ UCO/Lick Observatory and Department of Astronomy and \\ Astrophysics, University of California, Santa Cruz, CA 95064, USA
}

Ian Thompson

Observatories of the Carnegie Institute of Washington, 813 Santa

Barbara Street, Pasadena, CA 91101, USA

\begin{abstract}
We present a method to determine the star formation history (SFH) of a mixed stellar population, based on an iterative maximum Likelihood comparison of stellar photometry to model color-magnitude diagrams. We demonstrate the algorithm on a subregion of the Large Magellanic Cloud, observed as part of our ongoing Magellanic Clouds Photometric Survey. We will eventually perform this analysis on hundreds of regions in both Clouds, resulting in a homogeneous SFH map of these galaxies.
\end{abstract}

\section{Introduction}

The evolution of a galaxy is defined by its star formation history (SFH): the star formation rate as a function of time, position and metallicity throughout the galaxy. Understanding the detailed SFH of resolved local group galaxies can potentially lead to a greater understanding of a variety of astrophysical problems, including the evolution of distant, unresolved galaxies, the propagation of star formation, and the role of galactic gravitational interactions.

\section{Data and Method}

We have $U B V I$ photometry for approximately 57,000 stars in a randomly selected $24^{\prime} \times 24^{\prime}$ region of the Large Magellanic Cloud, from our ongoing Magellanic Clouds Photometric Survey (for details, see Zaritsky et al. 1997). The survey will eventually provide $U B V I$ photometry of tens of millions of stars in each of the Clouds.

We determine the SFH by matching the observed photometric distribution of stars as closely as possible using stellar evolutionary models. The Padua isochrones (Bertelli et al. 1994) are used because they provide wide coverage in age and metallicity. Every point along each isochrone is assigned a relative occupation probability (OP) based on a Salpeter IMF. The OP of each isochrone point is then distributed along the reddening line according to the observed red- 


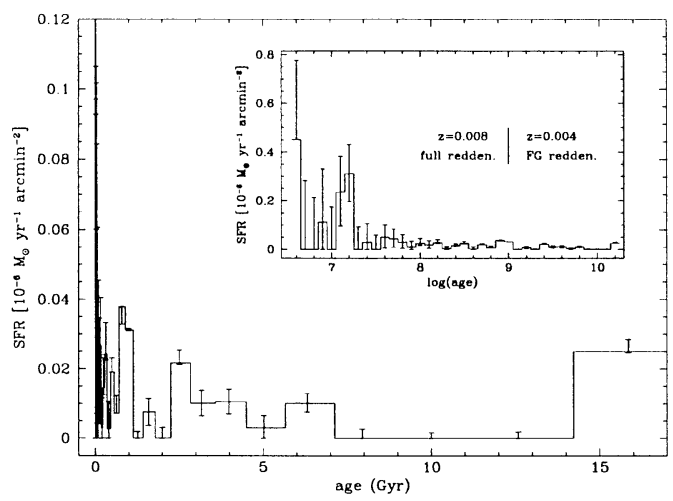

Figure 1. The derived SFH of the LMC region.

dening statistics (Harris et al. 1997) and error-blurred according to results from artificial star tests. These procsses result in a library of model Hess diagrams. One can then simply combine Hess diagrams linearly, assigning each an independent amplitude, to form a composite Hess diagram that can be compared to the data. The set of isochrone amplitudes describes the SFH of the model. To reduce the number of degrees of freedom in the model, we have imposed a chemical enrichment law, so that there is only one possible metallicity at each age. Eventually, we will allow metallicity choices at every epoch.

We use an "amoeba" algorithm to find the set of isochrone amplitudes that produces the best match to the observed Hess diagram. It starts at a randomly selected point in parameter space, and computes the Likelihood of the resulting model. It then takes a step in each parameter dimension, and by recomputing the Likelihood at these new locations, determines the local Likelihood gradient. A step in the direction of the gradient is taken, and the procedure iterates until a maximum Likelihood is found.

\section{Results}

The derived SFH of this region is shown in Figure 1, a plot of the isochrone amplitudes of the most likely model. It indicates an ancient burst of star formation, followed by $7 \mathrm{Gyr}$ of quiescence, followed by an epoch of increasing, more or less continuous star formation activity that is continuing today. The error bars indicate the $95 \%$ confidence limits on each model parameter, but we believe that these underestimate the true uncertainty associated with each amplitude.

\section{References}

Bertelli, G., et al. 1994, A\&AS, 106, 275

Harris, J., Zaritsky, D., \& Thompson, I. 1997, AJ, 114, 1933

Zaritsky, D., Harris, J., \& Thompson, I. 1997, AJ, 114, 1002 